\title{
BMJ Open Do lifestyle measures to counter COVID-19 affect frailty rates in elderly community dwelling? Protocol for cross- sectional and cohort study
}

\author{
Tomoyuki Shinohara (D , ${ }^{1}$ Kosuke Saida, ${ }^{1}$ Shigeya Tanaka, ${ }^{1}$ Akihiko Murayama ${ }^{2}$
}

To cite: Shinohara T, Saida K, Tanaka S, et al. Do lifestyle measures to counter COVID-19 affect frailty rates in elderly community dwelling?

Protocol for cross-sectional and cohort study. BMJ Open 2020;10:e040341. doi:10.1136/ bmjopen-2020-040341

- Prepublication history for this paper is available online. To view these files, please visit the journal online (http://dx.doi. org/10.1136/bmjopen-2020040341).

Received 11 May 2020

Revised 07 July 2020

Accepted 30 September 2020

Check for updates

(C) Author(s) (or their employer(s)) 2020. Re-use permitted under CC BY-NC. No commercial re-use. See rights and permissions. Published by BMJ.

${ }^{1}$ Department of Physical

Therapy, Faculty of Health Care, Takasaki University of Health and Welfare, Takasaki, Gunma, Japan

${ }^{2}$ Department of Physical

Therapy, Faculty of

Rehabilitation, Gunma University of Health and Welfare, Maebashi, Gunma, Japan

Correspondence to Dr Tomoyuki Shinohara; shinohara-t@takasaki-u.ac.jp

\section{ABSTRACT}

Introduction Local activities that functioned to prevent frailty in the elderly have been suspended or reduced as a countermeasure against COVID-19. As a result, frailty rates are expected to increase, and frailty is expected to worsen as a secondary problem associated with COVID-19 countermeasures. Therefore, this study aims to determine the extent of frailty in the elderly associated with lifestyle changes implemented as COVID-19 countermeasures, to ascertain actual lifestyle changes and clarify the existence of Corona-Frailty. We will also conduct Corona-Frailty screening to verify the effect of support provided as feedback to supporters of the elderly.

Methods and analysis The survey target area is Takasak City, Gunma Prefecture, Japan. Phase I aims to verify the short-term effects of COVID-19. A questionnaire will be distributed to 465 community-dwelling elderly people, and responses will be obtained by post. Frailty will be evaluated using the Frailty Screening Index. Respondents who are frail and have had many changes in their lifestyle will be screened as high-risk people, and feedback will be provided to local supporters. The aim of Phase II will be to verify the long-term effects of COVID-19 and the effect of screening. A similar survey will be distributed twice after the first survey, once after 6 months and again after 1 year and the frailty rate will be tested. Furthermore, out of the subjects identified with frailty in Phase I, the progress of those who were screened and those who were not screened will be compared between groups.

Ethics and dissemination This study has been approved by the Research Ethics Committee of the Takasaki University of Health and Welfare (approval number: 2009). The results of this study will be reported to the policymaker, presented at academic conferences and published in peer-reviewed journals.

Trial registration number UMIN000040335.

\section{INTRODUCTION}

\section{Background}

The novel COVID-19 first occurred in Wuhan, China, in November 2019. The Japanese government declared Japan's first state of emergency covering seven prefectures on 7 April, $^{1}$ which was extended to all 47 prefectures on 16 April. $^{2}$ The Japanese public were asked to avoid mass gatherings and to

\section{Strengths and limitations of this study}

- This study aims to clarify the increase in frailty rates among community-dwelling elderly as a secondary problem associated with COVID-19; that is, the aim is to clarify the existence of Corona-Frailty.

- This study will screen frail subjects whose lifestyle has been affected by COVID-19 measures and will clarify the effect of providing feedback to supporters of the elderly.

- There are almost no similar surveys at present because the pandemic is ongoing, and people's lives are yet to return to normal.

- For this study, the survey form will be distributed by local volunteers who will continue to provide support to the elderly, and the form will be collected by post.

- The subjects will be limited to community-dwelling elderly who receive support from local volunteers.

thoroughly implement social distancing as measures to prevent the spread of infection in Japan. As a result, activities involving elderly outings (kayoinoba) have been suspended, and support involving regular visits to the elderly has been reduced.

Kayoinoba is a setting where activities are available for people to participate in, which includes strength training and light exercises in groups, and is also a place where people can simply interact with each other. Regular visits to the elderly is a type of lifestyle support aimed at preventing anxiety and isolation among the elderly living alone. These are not public services; rather, they are activities run by local volunteers, ${ }^{3}$ and are important local activities that support the lives of elderly people. Japan is a super-ageing society, and a community-based integrated care system is being built to support elderly people who live in the community. ${ }^{45}$ Therefore, these local activities play a role in preventing frailty in the elderly.

Frailty is a condition where a person has increased vulnerability to stress due to a 
decline in their physiological reserve, which then tends to result in poor health. ${ }^{6}$ Frailty has three elements: physical, cognitive and social. A systematic review reported that $9.9 \%$ of the 44894 community-dwelling elderly experienced physical frailty. ${ }^{7}$ Cognitive frailty is a condition where a person displays a decline in both physical and cognitive functions, ${ }^{8}$ and in Japan, the rates of cognitive frailty have been reported as $4.4 \%$ in 594 communitydwelling elderly ${ }^{9}$ and $1.2 \%$ in 8864 people. ${ }^{10}$ Social frailty focuses on the social aspect of this condition and is defined by various elements, including a person's satisfaction with their social needs, social resources and social activities. The rate of social frailty in Japan was $10.2 \% .^{11}$

Frail people have an increased risk of falls, decline in activities of daily living ability, hospitalisation and death. ${ }^{6}$ This condition also affects mental function and is associated with an onset of depressive symptoms ${ }^{12}$ and cognitive decline. ${ }^{13}$ Social frailty without activities involving interaction with the local community worsens life prognosis, similar to physical frailty. ${ }^{14}$ Living alone and not leaving the house and/or interacting with other people also leads to a decline in physical function. ${ }^{11}$ The greater the availability of local activities such as kayoinoba and regular visits to the elderly, the lower the risk of the onset of frailty. ${ }^{15}$ It is said that social frailty leads to isolation and almost certainly results in physical frailty and sarcopenia too. ${ }^{16}$ Therefore, frailty is a major concern since it causes physical harm and results in psychological and/or social harm.

There is no doubt that the treatment and prevention of the spread of infection are the top priorities in the current situation with the pandemic still ongoing. However, it is easy to predict that the suspension of local activities will result in increased frailty rates and worsened frailty in the elderly. We hypothesise that frailty related to lifestyle changes implemented as COVID-19 countermeasures will develop as "Corona-Frailty' 17 and the frailty rate will be higher than before. There are also grave concerns about the elderly themselves and the supporters who provide support to the elderly. Preventive measures are needed to determine how the elderly can maintain their lifestyles without developing Corona-Frailty.

\section{Purpose}

Concerns have been raised regarding the increased mortality rate of COVID-19 in elderly people with respiratory disease and frailty, ${ }^{18}$ and the need for ageindependent evaluation of frailty in emergency medical settings during pandemics has been proposed. ${ }^{19}$ However, we were unable to find any reports of the effects of COVID-19 on frailty in the elderly.

It has now been more than 2 months since the suspension of local activities that fulfilled a role in preventing frailty in community-dwelling elderly. Thus, we planned this study to achieve the following aims:

- Clarify the actual frailty condition of communitydwelling elderly people who are subject to lifestyle restrictions due to COVID-19 measures for determining the existence of Corona-Frailty.

- Clarify the longitudinal reality based on predicting the long-term effect of COVID-19.

- Screen frail elderly people whose lifestyle has been affected by COVID-19 measures, provide feedback on the associated risk of frailty to supporters of elderly people and clarify the effectiveness of this feedback.

\section{TRIAL DESIGN AND METHODS \\ Study design}

Phase I: A cross-sectional survey will be distributed to investigate the condition of frailty in community-dwelling elderly whose lives are affected by the COVID-19 measures (figure 1). This will be conducted to verify the short-term effects of COVID-19.

Phase II: A prospective cohort study will be conducted, investigating the subjects surveyed in Phase I (figure 1). This will be carried out to verify the long-term effects of COVID-19 and the effect of screening support provided to frail subjects whose lifestyles had changed.

\section{Setting}

This study will be conducted in Takasaki City, Gunma Prefecture. The proportion of the population over the age of 65 in Japan was $28.4 \%$ in 2019 (95\% CI 29.2 to 30.9 among 47 prefectures in Japan), ${ }^{20}$ and it is estimated to

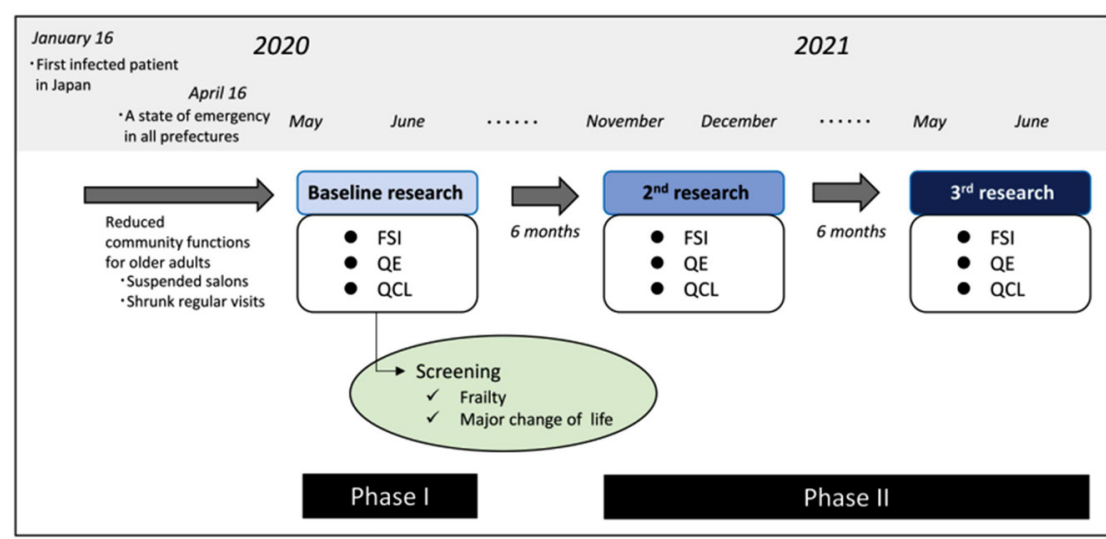

Figure 1 Overview of study design. FSI, Frailty Screening Index; QCL, Questionnaire for Change of Life; QE, Questionnaire for the Elderly. 
increase to $30.0 \%$ in $2025 .^{21}$ The proportion of the population over the age of 65 in the study area was $28.3 \%$ in 2019 (95\% CI 32.3 to 38.8 among 35 municipalities in Gunma Prefecture), ${ }^{22}$ and is estimated to be $30.8 \%$ in $2025 .^{23}$ The target area of this study is an area that roughly follows the national average of Japan, and can therefore be regarded as an average area in Japan in terms of the population ratio of the elderly.

\section{Participants}

\section{Inclusion criteria}

The study will target elderly people aged 65 and over who live in local housing rather than in hospitals or aged care facilities. Subjects will not be excluded due to the presence of specific types of comorbidities and/or disorders, to enable a broad investigation of the actual condition of community-dwelling elderly.

\section{Exclusion criteria}

The subjects will have the choice to withdraw consent. If they wish to withdraw consent, they need to contact the consent withdrawal contact person. The fact that consent can be withdrawn and the contact details for withdrawing consent will be specified during the distribution of the survey form. The instructions will also state that consent cannot be withdrawn after the study results have been presented at academic conferences and/or published in journals.

\section{Outcome measures}

Primary outcome measures

Frailty and prefrailty will be evaluated using the Frailty Screening Index (FSI) (table 1) developed by Yamada and $\mathrm{Arai}^{24}$ based on the frailty evaluation criteria proposed by Freid $e t a l^{6}$ The FSI was used to assess physical/psychological frailty and had predictive validity for disability ${ }^{24}$ and concurrent validity for social frailty ${ }^{25}$ among Japanese elderly. The FSI is a questionnaire that comprises five items answered with simple Yes/No responses, and scoring ranging from 0 to 5 . It does not require an actual measurement of grip strength or walking speed. The frailty status was based on the participant's score, where a

\section{Table 1 Frailty Screening Index}

\begin{tabular}{llll}
\hline & & \multicolumn{2}{l}{ Score } \\
\cline { 2 - 4 } No & Item & Yes & No \\
\hline (1) & $\begin{array}{l}\text { Have you lost 2 kg or more in the past 6 } \\
\text { months? }\end{array}$ & 1 & 0 \\
(2) Do you think you walk slower than before? & 1 & 0 \\
(3) Do you go for a walk for your health at & 0 & 1 \\
& $\begin{array}{l}\text { least once a week? } \\
\text { (4) } \quad \text { Can you recall what happened 5 min ago? }\end{array}$ & 0 & 1 \\
(5) $\quad \begin{array}{l}\text { In the past 2 weeks, have you felt tired } \\
\quad \text { without a reason? }\end{array}$ & 1 & 0 \\
\hline
\end{tabular}

A subject with total scores of 3 or more was defined as frail, 1-2 as prefrailty and 0 as robust. score of 3 or more was defined as frailty, 1-2 as prefrailty and 0 as robust. ${ }^{24}$

\section{Secondary outcome measures}

The questionnaire for the elderly (QE, table 2$),{ }^{26}$ prepared by the Japanese Ministry of Health, Labour and Welfare, will be used to comprehensively evaluate the health status of the subjects based on the characteristics of the elderly. In Japan, the use of QE has been required to ascertain the condition of elderly people aged 75 and over during medical examinations, since April 2020. The questionnaire comprises 10 different areas: (1) health condition, (2) mental health condition, (3) eating habits, (4) oral function, (5) weight change, (6) exercise/falls, (7) cognitive function, (8) smoking, (9) social participation, and (10) social support. In consideration of the burden on the elderly, only 15 questions were included in the questionnaire. We will indicate social isolation, which became a critical factor as a result of the lifestyle restrictions due to the COVID-19 measures using the questionnaire items (9) social participation and (10) social support.

The Questionnaire for Change of Life (QCL) will be used to evaluate the impact of COVID-19 measures on daily life (table 3). The QCL was created referencing the Impact of Event Scale-Revised (IES-R), ${ }^{27}$ which has been translated into many languages. The reliability and validity of the IES-R has been verified as an Impact of Event Scale ${ }^{28}$ for evaluating psychological reactions caused by stressors such as pandemics and disasters. The QCL comprises five items related to frailty ${ }^{6111215}$ : physical activity, muscle strength, meal size, anxiety and communication opportunities, using 5-point Likert scale response options based on the IES-R. The participants will be asked about subjective changes in the past month to evaluate the changes in the person's lifestyle due to the measures implemented to prevent the spread of infection. Because we do not have the subject's condition prior to the study period, we will use the QCL to assess the current situation.

\section{Procedure}

Phase I: The survey method will involve the distribution of the questionnaire by local volunteers who run the kayoinoba and provide support with regular visits to elderly residents. The people who distribute the survey form will be defined as research collaborators. In addition to the survey form, we will also distribute the instructions for this study, a return envelope and a pamphlet to raise awareness of frailty prevention during lifestyle restrictions associated with COVID-19 measures. To maintain social distance as an infection control measure, the research collaborator's role is to distribute a set of survey forms. Once the person has received the survey form from the research collaborator, they will be candidates for this study. If the person agrees to participate in this study, they will write their name on the survey form and return the form by post. Any person who completes these procedures will be a subject in this study. Researchers will be responsible for the cost of the postage and envelopes. 
Table 2 Questionnaire for the Elderly

\begin{tabular}{|c|c|c|c|c|}
\hline No & Type & Item & \multicolumn{2}{|c|}{ Response } \\
\hline (1) & Health condition & How is your health? & \multicolumn{2}{|c|}{$\begin{array}{l}\text { 1. Good } \\
\text { 2. Fairly good } \\
\text { 3. Normal } \\
\text { 4. Not very good } \\
\text { 5. Bad }\end{array}$} \\
\hline (2) & $\begin{array}{l}\text { Mental health } \\
\text { condition }\end{array}$ & Are you satisfied with your daily life? & \multicolumn{2}{|c|}{$\begin{array}{l}\text { 1. Satisfied } \\
\text { 2. Somewhat satisfied } \\
\text { 3. Somewhat dissatisfiec } \\
\text { 4. Dissatisfied }\end{array}$} \\
\hline (3) & Eating habits & Do you consistently eat three meals a day? & Yes & No \\
\hline (4) & Oral function & $\begin{array}{l}\text { Has it become more difficult to eat hard food than it was } 6 \\
\text { months ago? }\end{array}$ & Yes & No \\
\hline (8) & \multirow{2}{*}{ Exercise/falls } & Have you fallen in the past year? & Yes & No \\
\hline (9) & & Do you take a walk to exercise at least once a week? & Yes & No \\
\hline (10) & \multirow[t]{2}{*}{$\begin{array}{l}\text { Cognitive } \\
\text { function }\end{array}$} & $\begin{array}{l}\text { Are you told that you are forgetful, with comments such as } \\
\text { 'you are always telling me the same thing'? }\end{array}$ & Yes & No \\
\hline (11) & & Do you sometimes forget what day and month it is that day? & Yes & No \\
\hline (12) & Smoking & Do you smoke cigarettes? & \multicolumn{2}{|c|}{$\begin{array}{l}\text { 1. Yes, I smoke. } \\
\text { 2. No, I do not smoke. } \\
\text { 3. I quit smoking. }\end{array}$} \\
\hline (13) & \multirow{2}{*}{$\begin{array}{l}\text { Social } \\
\text { participation }\end{array}$} & Do you go out at least once a week? & Yes & No \\
\hline (14) & & Are you normally in close contact with family and friends? & Yes & No \\
\hline
\end{tabular}

Using the returned survey form, any respondent who was defined as frail based on FSI and who responded with 'decreased' for QCL questions on physical activity, muscle strength, meal size and communication opportunities or 'increased' for anxiety, for a total of 2 or more items, will be screened as high risk (figure 1) and that

\begin{tabular}{|lll|}
\hline \multicolumn{3}{l}{ Table 3 Questionnaire for change of life } \\
\hline No. & Item & Response \\
\hline (1) & $\begin{array}{l}\text { Amount of daily } \\
\text { movement }\end{array}$ & $\begin{array}{l}\text { Decreased / Slightly decreased / } \\
\text { Unchanged / Slightly increased / } \\
\text { Increased }\end{array}$ \\
(2) $\quad$ Leg muscle & $\begin{array}{l}\text { Weaker / Slightly weaker / } \\
\text { Unchanged / Slightly stronger / } \\
\text { Strength }\end{array}$ & $\begin{array}{l}\text { Decreased / Slightly decreased / } \\
\text { Unchanged / Slightly increased / } \\
\text { Increased }\end{array}$ \\
(3) $\quad$ Meal size & Decreased / Slightly decreased / \\
(4) Worry or anxiety & $\begin{array}{l}\text { Unchanged / Slightly increased / } \\
\text { Increased }\end{array}$ \\
(5) $\quad$ Opportunities of & $\begin{array}{l}\text { Decreased / Slightly decreased / } \\
\text { talking to people } \\
\text { Increased / Slightly increased / }\end{array}$ \\
\hline
\end{tabular}

result will be provided as feedback to the research collaborators. It is expected that understanding these risks will enable research collaborators, namely, the supporters, to enhance their support for regular visits to the elderly and provide lifestyle support, increase family support and assist in the introduction and deployment of public services.

Phase II: The subjects in Phase I were surveyed again with a similar survey in Phase II, which will be distributed and collected at intervals of 6 months and 1 year after Phase I (figure 1). If the Japanese government's request to avoid mass gatherings and to thoroughly implement social distancing as an infection control measure is protracted, we might consider shortening the 6 months to 3 or 4 months due to the negative impact on frailty.

\section{Patient and public involvement}

Participants were not involved in the development of the research question nor study design. However, the research collaborators at a Public Interest Incorporated and Social Welfare Corporations were involved in coordinating with local volunteers. The local volunteers who were going to distribute the survey form were involved in study design. 


\section{Data management}

We will collect data manually from the survey form, which is returned with the participant's consent signature. To avoid generating several errors, we will run a double check on the input data for $10 \%$ of the total reported data. The data will be deidentified and a master linking list with identities will be kept and stored separately from the data. Data will be stored on an external storage medium in a locked cabinet in a secure location. Access to the returned survey form and data will be limited to the study personnel.

\section{Statistical methods}

\section{Sample size}

The sample size required for this study was estimated based on the n:p rule of thumb. ${ }^{29}$ The $n: p$ is the minimum recommended ratio, $(n)$ is the sample size and $(p)$ is the number of variables being analysed. The n:p ratio recommended is at least $10 .{ }^{30}$ The FSI has 5 items, the QE has 15 items and the QCL has 5 items. The total number of variables is 25 . If $n: p$ is set at 10 , the minimum sample size is 250. We plan for the forms to be returned by post in this study, and the review results on the recovery rate for similar studies in Japan illustrated that the recovery rate for studies on elderly frailty, health and lifestyle using the postal method over the past 3 years was $67.8 \%-76.9 \% .^{31-34}$ Based on these findings, we estimated the recovery rate of the survey form in this study to be $60 \%$, and incomplete survey forms to be $10 \%$. Therefore, we ultimately plan to distribute 465 survey forms.

\section{Analysis}

In Phase I, we will clarify the actual effect of the COVID-19 countermeasures in daily life. The frailty and prefrailty rates will be calculated using FSI. The reference prevalence rates of frailty, prefrailty and robust status, based on a meta-analysis in Japan, were $7.5 \%, 48.1 \%$ and $44.4 \%$, respectively. ${ }^{35}$ These will be used as a reference value and the difference in the proportion of the frailty rate in this study will be verified using the $\mathrm{Z}$ test, to clarify whether frailty has increased due to the impact of COVID-19, that is, whether Corona-Frailty occurred. Descriptive statistics for gender and age will be performed to test the association with frailty status. We will also collate the answers to 10 types of QE and five QCL items to ascertain the health condition of the subjects. We will cross-tabulate each of the QCL results for frailty status and verify the findings using the $\chi^{2}$ test or Fisher's exact test to determine the relationship between the change in daily life due to COVID-19 measures and frailty status.

Phase II will clarify the long-term effects of COVID-19 using Phase I as a baseline. The difference in the ratio of the frailty rate and the prefrailty rate at intervals of 6 months and 1year after the baseline will be verified using the $\mathrm{Z}$ test. Ten types of QE and five QCL items will be collated, and the difference at each time point will be verified using the $\chi^{2}$ test or Fisher's exact test. To indicate the factors that contribute to the transition to frailty, multiple logistic regression analysis based on forced entry methods will be used. The transition to frailty or the nontransition to frailty is the dependent variable, and each item of QE and QCL are the independent variables. Of the subjects who were frail during Phase I, the frailty rates of the screened group and the non-screened group in 6 months and 1 year after the baseline will be examined to clarify the effect of Corona-Frailty screening support. The comparison will be verified using the $\mathrm{Z}$ test. Furthermore, we will apply two types of analysis: screening support and intention-to-treat analysis based on the screened group and the non-screened group.

\section{ETHICS AND DISSEMINATION \\ Ethics}

This study will commence once it has been approved by the Research Ethics Committee of the Takasaki University of Health and Welfare (approval number 2009). The study will be conducted in accordance with the tenets of the Declaration of Helsinki. The purpose of this study and the handling of information will be explained to all subjects, and a signed written consent will be obtained.

\section{Dissemination}

This study aims to clarify the effect of the unprecedented COVID-19 on frailty in community-dwelling elderly. It is expected to have both short-term and long-term effects. The policymaker has started to implement measures in view of the impact of COVID-19 on the elderly. The results of this study will be reported to the policymaker so that they can be used as materials for future measures. In particular, the results of Phase I, which will evaluate the short-term effects of COVID-19, including the existence of Corona-Frailty, will be communicated in the preliminary findings report. In addition, the activity details and study results will be presented at academic conferences and published in peer-reviewed journals.

\section{DISCUSSION}

We assume that the frailty rate of the study participants in Phase I will be higher than the reference rate. This is because Phase I research was conducted during the 2 months after the introduction of preventive measures against COVID-19 that resulted in the suspension or reduction of the local activities that functioned to prevent frailty in the elderly. If the frailty rate increases, it may be regarded as Corona-Frailty. We suggest that this study's target area is regarded as a representative sample area in Japan in terms of the population ratio of the elderly, as there were no contradictions in our comparison with the historical data in Japan.

Currently, there are few studies on secondary problems associated with COVID-19 because the focus is on the treatment and prevention of the spread of infection. Social distancing measures are required as a countermeasure against infectious diseases, ${ }^{36}$ and it is necessary to pay 
attention to research methods. Zhang and $\mathrm{Ma}^{37}$ reported the impact of COVID-19 on mental health and quality of life, evaluated using the Impact of Event Scale. ${ }^{38}$ This survey was conducted via the internet, and the mean age of the subjects was young at 38 years; therefore, it does not indicate the reaction of the elderly. The internet usage rate of the elderly in Japan is $45.8 \%$ in households with only elderly occupants. ${ }^{39}$ In recent years, the share of smartphones has become larger than that of personal computers. The smartphone usage rate was reported to be $39 \%$ for people aged between 65 and 74 years, and $15 \%$ for those aged 75 and above, ${ }^{40}$ and intervention studies on elderly people using smartphones as assistive devices have been conducted. ${ }^{41}$ However, when internet and smartphone usage rates are less than half, it is not suitable to use these methods to survey the actual situation in an area. Wolf $e t a l^{42}$ have conducted a telephone survey of the awareness and attitude towards COVID-19 in young and old people. In a pandemic situation, it is essential to consider how best to access the target population. In this study, we were able to plan the distribution of the survey form by using the limited available access because local volunteers continue to check in on the elderly. We believe that this can provide a feasible method for conducting a survey in a pandemic situation.

One of the aims of this study is to verify the effect of support using Corona-Frailty screening. Kuwahara $e^{t} a t^{43}$ stated the need for practical information to properly implement local activities based on the cancellation of local activities, and argued that isolation in the local area is a particularly major issue. Armitage and Nellums ${ }^{44}$ also stated that although isolation is essential and effective in preventing infection, secondary problems do develop due to isolation. However, the WHO response to various problems such as exacerbation of symptoms and depression in the elderly is not sufficient. ${ }^{45}$ Yang $e t a l^{46}$ proposed the need for adequate mental support for depressive symptoms during the period of isolation and restricted movement associated with COVID-19 infection control. Frailty has three elements: physical, cognitive and social, all of which are affected by COVID-19. We hope that effective support will be deployed by performing Corona-Frailty screening at an early stage and providing information to supporters.

This study has several limitations. First, the FSI has insufficient validity for other frailty criteria. However, to maintain the implementation of social distancing as a preventive measure against infection, we had to use an easy questionnaire that the elderly could answer without the support of others at home. Second, we could not research the health and frailty status of the participants as a reference condition before the pandemic. Third, we have set a wide inclusion to enable a broad investigation of the actual condition and do not collect the names of the diseases. This study has been conducted under limited conditions while taking infection control measures.

Frailty increases the risk of hospitalisation and admission to aged care facilities, ${ }^{47}$ as there is a large degree of functional deterioration, even if it is transient, and frailty diminishes a person's ability to recover to their original condition. ${ }^{48}$ Gill et at $t^{49}$ reported that physical frailty increases the risk of decline in life function, persistent decline in life function and decline in life function associated with admission to aged care facilities. On the other hand, appropriate intervention for individuals with frailty can be expected to improve their physical function and enable them to break away from frailty. ${ }^{50}$ It is necessary to establish a system that is able to provide prompt support by properly evaluating the increase in frailty rates and deterioration of frailty due to the impact of COVID-19. COVID-19 continues to spread, and there is an urgent need to adopt measures for the elderly who are particularly susceptible. Research to resolve secondary problems such as Corona-Frailty and prompt disclosure of information are expected.

Acknowledgements We would like to express our sincere gratitude to Kenichi Sudo, Munehisa Sudo, Professor Atsushi Kuwabara, and Professor Satoshi Tanaka of the Takasaki University of Health and Welfare; Chieko Mesaki of the Sawayaka Welfare Foundation; Junko Ishii, Kumi Aoki and Norie Torizuka of the Social Welfare Corporation Kibokan; Miyuki Ogawa of the Social Welfare Corporation Shinmachi Genkimura; Yumi Ino of the Social Welfare Corporation Ninosawa Shinpukukai; Seiichi Asanuma of the Social Welfare Corporation Turbulence Fukushikai; and Ayako Yamazaki of the Nishi-Agatsuma Welfare Hospital. We would also like to thank the Takasaki-shi district welfare commissioners Susumu Shimomura, Shizuko Nakajima,Noriko Takizawa, Misao Shiraishi, Tomiko Takeuchi, Masae Ozawa, Tamotsu Nakamura, Tadaaki Nakazawa, Yasuo Nagasaka, Toshiko Nakazawa, Setsuko Okada, Noriko Umeki, Takeko Kosone, Nobuko Kaseda, Kazuaki Kuwabara, Yuriko Yoshiara, Ryo Koike, Masaaki Arai. We would like to thank Editage (www. editage.com) for free English language editing for supporting COVID-19 research.

Contributors The research question was proposed by TS and KS, and planning was performed by TS, KS, ST and AM. The protocol manuscript was prepared by TS, KS and ST. TS and KS coordinated with research collaborators, and TS, KS, ST and AM collated and analysed the study data and presented the research results. All authors contributed to the writing and critical revision of the manuscript and approved the final manuscript.

Funding The parts of this work are supported by the Nippon Life Insurance Foundation (Grant 2020-0203-04) and the Japanese Society for the Promotion of Science KAKENHI (Grant 19K19712).

Competing interests None declared.

Patient and public involvement Patients and/or the public were involved in the design, or conduct, or reporting, or dissemination plans of this research. Refer to the Methods section for further details.

Patient consent for publication Obtained.

Provenance and peer review Not commissioned; externally peer reviewed.

Open access This is an open access article distributed in accordance with the Creative Commons Attribution Non Commercial (CC BY-NC 4.0) license, which permits others to distribute, remix, adapt, build upon this work non-commercially, and license their derivative works on different terms, provided the original work is properly cited, appropriate credit is given, any changes made indicated, and the use is non-commercial. See: http://creativecommons.org/licenses/by-nc/4.0/.

ORCID iD

Tomoyuki Shinohara http://orcid.org/0000-0002-4213-6841

\section{REFERENCES}

1 Looi M-K. Covid-19: Japan declares state of emergency as Tokyo cases SOAR. BMJ 2020;369:m1447.

2 Looi M-K. Covid-19: Japan prepares to extend state of emergency nationwide as "untraceable" cases soar. BMJ 2020;369:m1543. 
3 Tsutsui T. Implementation process and challenges for the community-based integrated care system in Japan. Int J Integr Care 2014;14:e002.

4 mhlw. Ministry of health, labour and welfare. Available: https:// www.mhlw.go.jp/file/06-Seisakujouhou-12300000-Roukenkyoku/ 0000075982.pdf [Accessed 16 Apr 2020].

5 mhlw. Ministry of health, labour and welfare. Available: https://www. mhlw.go.jp/english/policy/care-welfare/care-welfare-elderly/dl/ establish_e.pdf [Accessed 16 Apr 2020].

6 Fried LP, Tangen CM, Walston J, et al. Frailty in older adults: evidence for a phenotype. J Gerontol A Biol Sci Med Sci 2001;56:M146

7 Collard RM, Boter H, Schoevers RA, et al. Prevalence of frailty in community-dwelling older persons: a systematic review. J Am Geriatr Soc 2012;60:1487-92.

8 Kelaiditi $E$, Cesari M, Canevelli M, et al. Cognitive frailty: rational and definition from an (I.A.N.A./I.A.G.G.) international consensus group. $J$ Nutr Health Aging 2013:17:726-34.

9 Roppolo M, Mulasso A, Rabaglietti E. Cognitive frailty in Italian community-dwelling older adults: prevalence rate and its association with disability. J Nutr Health Aging 2017;21:631-6.

10 Shimada H, Makizako H, Lee S, et al. Impact of cognitive frailty on daily activities in older persons. J Nutr Health Aging 2016;20:729-35.

11 Makizako $\mathrm{H}$, Shimada $\mathrm{H}$, Tsutsumimoto $\mathrm{K}$, et al. Social frailty in community-dwelling older adults as a risk factor for disability. J Am Med Dir Assoc 2015;16:1003:e7-11.

12 Makizako H, Shimada H, Doi T, et al. Physical frailty predicts incident depressive symptoms in elderly people: prospective findings from the Obu study of health promotion for the elderly. J Am Med Dir Assoc 2015;16:194-9.

13 Samper-Ternent R, Al Snih S, Raji MA, et al. Relationship between frailty and cognitive decline in older Mexican Americans. J Am Geriatr Soc 2008;56:1845-52.

14 Garre-Olmo J, Calvó-Perxas L, López-Pousa S, et al. Prevalence of frailty phenotypes and risk of mortality in a community-dwelling elderly cohort. Age Ageing 2013;42:46-51.

15 Okura M, Ogita M, Yamamoto M, et al. Community activities predict disability and mortality in community-dwelling older adults. Geriatr Gerontol Int 2018;18:1114-24.

16 Tanaka T, Takahashi K, Akishita M, et al. Social participation for flail prevention: the impact of social flail. Geriatric Medicine 2017:55:159-63.

17 Shinohara T, Saida K, Tanaka S, et al. Rapid response: impact of the COVID-19 pandemic on frailty in the elderly citizen; corona-frailty. BMJ 2020;369:m1543.

18 Bonanad C, García-Blas S, Tarazona-Santabalbina FJ, et al. Coronavirus: the geriatric emergency of 2020. joint document of the geriatric cardiology section of the Spanish Society of cardiology and the Spanish Society of geriatrics and gerontology. Rev Esp Cardiol 2020

19 Boreskie KF, Boreskie PE, Melady D. Age is just a number - and so is frailty: Strategies to inform resource allocation during the COVID-19 pandemic. CJEM 2020;22:411-3.

20 Statistics Bureau of Japan. Current population estimates as of October 1 2019, 2019. Available: https://www.stat.go.jp/english/data/ jinsui/2019np/index.html [Accessed 21 Apr 2020].

21 Cabinet Office Japan. Annual Report on the Ageing Society [Summary] FY, 2019. Available: https://www8.cao.go.jp/kourei/ english/annualreport/2019/pdf/2019.pdf [Accessed 20 Apr 2020]

22 Gunma prefecture. Results of Gunma Prefecture demographic survey by age, 2019. Available: https://toukei.pref.gunma.jp/nbj/data/ nbj2019.xls [Accessed 30 Jun 2021].

23 Takasaki City. Koreicha Anshin plan future estimates in 2025, 2018. Available: http://www.city.takasaki.gunma.jp/docs/2014011700659/ files/2025keniki.pdf [Accessed 21 Apr 2020].

24 Yamada M, Arai $\mathrm{H}$. Predictive value of frailty scores for healthy life expectancy in community-dwelling older Japanese adults. J Am Med Dir Assoc 2015;16:1002:e7-11.

25 Yamada M, Arai H. Social frailty predicts incident disability and mortality among community-dwelling Japanese older adults. J Am Med Dir Assoc 2018;19:1099-103.

26 mhlw. Ministry of health, labour and welfare. Available: https://www. mhlw.go.jp/content/12401000/000557576.pdf [Accessed 25 Apr 2020].

27 Weiss DS, Marmar CR. The impact of event scale - revised. In: Wilson JP, Keane TM, eds. Assessing psychological trauma and PTSD. New York: Guilford Press, 1997: 399-411.
28 Horowitz M, Wilner N, Alvarez W. Impact of event scale: a measure of subjective stress. Psychosom Med 1979;41:209-18.

29 MacCallum RC, Widaman KF, Zhang S, et al. Sample size in factor analysis. Psychol Methods 1999;4:84-99.

30 Everitt BS. Multivariate analysis: the need for data, and other problems. Br J Psychiatry 1975;126:237-40.

31 Sugiura K, Hayachi C, Yokojima K, et al. Declining Oral Functioning's Effects on the Frequency of Leaving One's Home: Examining Individuals Living in the Community Who Require Minor Nursing Support. Jpn Med Assoc J 2018;26:232-40.

32 Seino S, Kitamura A, Tomine Y, et al. A community-wide intervention trial for preventing and reducing frailty among older adults living in metropolitan areas: design and baseline survey for a study integrating participatory action research with a cluster trial. $J$ Epidemiol 2019;29:73-81.

33 Ishikawa M, Yokoyama T, Takemi Y, et al. Association between satisfaction with state of health and meals, physical condition and food diversity, health behavior, and perceptions of Shopping difficulty among older people living alone in Japan. J Nutr Health Aging 2017;21:514-20.

34 Yamada $\mathrm{Y}$, Nanri $\mathrm{H}$, Watanabe $\mathrm{Y}$, et al. Prevalence of frailty assessed by fried and Kihon checklist indexes in a prospective cohort study: design and demographics of the Kyoto-Kameoka longitudinal study. J Am Med Dir Assoc 2017;18:733.e7-733.e15.

35 Kojima G, lliffe S, Taniguchi Y, et al. Prevalence of frailty in Japan: a systematic review and meta-analysis. J Epidemiol 2017;27:347-53.

36 World Health Organization. Responding to community spread of COVID-19, 2020. Available: https://www.who.int/publications-detail/ responding-to-community-spread-of-covid-19 [Accessed $25 \mathrm{Apr}$ 2020].

37 Zhang Y, Ma ZF. Impact of the COVID-19 pandemic on mental health and quality of life among local residents in Liaoning Province, China: a cross-sectional study. Int J Environ Res Public Health 2020;17:ijer ph17072381.

38 Lau JTF, Yang X, Tsui HY, et al. Positive mental health-related impacts of the SARS epidemic on the general public in Hong Kong and their associations with other negative impacts. J Infect 2006;53:114-24.

39 Statistics of Japan. Communication usage trend survey, 2018. Available: https://www.e-stat.go.jp/stat-search/files?page $=1 \&$ layout= datalist\&toukei $=00200356 \&$ tstat $=000001129917 \&$ cycle $=0 \&$ year $=$ $20180 \&$ month $=0 \&$ tclass $1=000001129920 \&$ stat infid $=000031831224$ [Accessed 25 Apr 2020].

40 Ofcom. Rise of the social seniors revealed, 2017. Available: https:// www.ofcom. org. uk/ about- ofcom/ latest/ media/ media- releases/ 2017/ risesocial-seniors[Accessed 23 Apr 2020].

41 Hawley-Hague $\mathrm{H}$, Tacconi $\mathrm{C}$, Mellone S, et al. Can smartphone technology be used to support an effective home exercise intervention to prevent falls amongst community dwelling older adults?: the together feasibility RCT study protocol. BMJ Open 2019;9:e028100.

42 Wolf MS, Serper M, Opsasnick L, et al. Awareness, attitudes, and actions related to COVID-19 among adults with chronic conditions at the onset of the U.S. outbreak: a cross-sectional survey. Ann Intern Med 2020;173:100-9.

43 Kuwahara K, Kuroda A, Fukuda Y. COVID-19: active measures to support community-dwelling older adults. Travel Med Infect Dis 2020;36:101638.

44 Armitage R, Nellums LB. COVID-19 and the consequences of isolating the elderly. Lancet Public Health 2020;5:e256.

45 Lloyd-Sherlock PG, Kalache A, McKee M, et al. WHO must prioritise the needs of older people in its response to the covid-19 pandemic. BMJ 2020;368:m1164

46 Yang Y, Li W, Zhang Q, et al. Mental health services for older adults in China during the COVID-19 outbreak. Lancet Psychiatry 2020;7:e19

47 Gill TM, Gahbauer EA, Han L, et al. The relationship between intervening hospitalizations and transitions between frailty states. $J$ Gerontol A Biol Sci Med Sci 2011;66:1238-43.

48 Clegg A, Young J, lliffe S, et al. Frailty in elderly people. Lancet 2013;381:752-62.

49 Gill TM, Allore HG, Holford TR, et al. Hospitalization, restricted activity, and the development of disability among older persons. JAMA 2004;292:2115-24.

50 Fairhall N, Sherrington C, Kurrle SE, et al. Effect of a multifactorial interdisciplinary intervention on mobility-related disability in frail older people: randomised controlled trial. BMC Med 2012:10:120. 